\title{
Evidence for superconductivity and a pseudogap in the new magnetic compound $\operatorname{PrAg}_{6} \mathrm{In}_{6}$
}

\author{
V.M. Dmitriev ${ }^{1,2,3}$, L.F. Rybaltchenko ${ }^{1,2}$, P. Wyder ${ }^{1}$, A.G.M. Jansen ${ }^{1}$, \\ N.N. Prentslau ${ }^{2}$, and W. Suski ${ }^{3,4}$ \\ ${ }^{1}$ Grenoble High Magnetic Field Laboratory, Max-Planck-Institut für Festkörperforschung and \\ Centre National de la Recherche Scientifique, B.P. 166, F-38042 Grenoble Cedex 9, France
}

\author{
${ }^{2}$ B. Verkin Institute for Low Temperature Physics and Engineering \\ of the National Akademy of Sciences of Ukraine, 47 Lenin Ave., Kharkov 61103, Ukraine \\ E-mail: dmitriev@ilt.kharkov.ua
}

${ }^{3}$ International Laboratory of High Magnetic Fields and Low Temperatures, 53-421 Wroclaw, Poland

${ }^{4}$ W. Trzebiatowski Institute of Low Temperatures and Structure Research, Polish Academy of Sciences, 50-950 Wroclaw, Poland

\begin{abstract}
Direct evidence for superconductivity in the new magnetic compound $\operatorname{PrAg}_{6} \operatorname{In}_{6}$ is revealed for the first time. The distinct Andreev-reflection current is observed in metallic point contacts (PC) based on this compound. The data obtained provide reason enough to suggest that the rise of superconductivity strongly depends on the local magnetic order varying over the sample volume. The triangular-shaped PC spectra $(d V / d I(V))$ in the vicinity of the zero-bias voltage suggest an unconventional type of superconducting pairing. As follows from the temperature and magnetic field dependences of the PC spectra, the superconducting energy gap structure transforms into the pseudogap one as the temperature or the magnetic field increases.
\end{abstract}

PACS: 74.70.Ad, 74.80.Fp

According to the conventional views, only a perfect antiferromagnetic (AFM) order is well compatible with superconductivity in a quite broad temperature range. New magnetic superconductors whose magnetic structures are far from having perfect AFM order provide a new insight into the problem of interplay between magnetism and superconductivity. A few years ago, a new class of magnetic superconductors with the $\mathrm{ThMn}_{12}$-type crystal structure was perceived to exist. Radio-frequency impedance and heat capacity measurements carried out on several compounds of this family have revealed distinct features in the corresponding characteristics which might be associated with superconductivity in some regions of the samples. Such indications of superconductivity were found in $\mathrm{LuFe}_{4} \mathrm{Al}_{8}, \mathrm{ScFe}_{4} \mathrm{Al}_{8}, \mathrm{YCr}_{4} \mathrm{Al}_{8}, \mathrm{YFe}_{4} \mathrm{Al}_{8}$, and $\operatorname{PrAg}_{6} \operatorname{In}_{6}[1,2]$. These compounds crystalize with the comparatively simple tetragonal body-centered structure of space group $I 4 / \mathrm{mmm}\left(D_{4 h}^{17}, \mathrm{nr}\right.$. 139). In spite of the AFM transition well above $100 \mathrm{~K}$, the magnetic structure remains very complicated [3,4].
So far, only very restricted information about the electronic structure is available [5]. Recently [6], we have proved an existence of a superconducting phase in $\mathrm{YFe}_{4} \mathrm{Al}_{8}$ by the point-contact (PC) Andreev-reflecton technique. The character of the measured PC spectra (differential resistance vs voltage, $d V / d I(V))$ implied an unconventional type of superconductivity in this compound.

Here, we present for the first time the direct evidence for superconductivity found in Andreev-reflection experiments on another recently synthesized compound $\operatorname{PrAg}_{6} \operatorname{In}_{6}$ [7]. This fact seems to be very impressive, because the Pr component is very antagonistic to superconductivity and can destroy it entirely. This is what occurs in $\mathrm{PrBa}_{2} \mathrm{Cu}_{3} \mathrm{O}_{x}$ unless special preparation techniques are used. We studied point contacts with the metallic conductivity of the needle-anvil geometry between Ag and freshly fractured surfaces of the $\operatorname{PrAg}_{6} \mathrm{In}_{6}$ polycrystal prepared by arc melting. According to the standard $x$-ray examination, the sample had the proper $\mathrm{ThMn}_{12}$-type lattice 
without noticeable traces of additional phases. The PC method employed enables one to measure on $\mathrm{N}-\mathrm{S}$ contacts the Andreev-reflection current whose energy and temperature dependences allow to infer the basic superconducting characteristics. The high reliability of PC Andreev-reflection method can be seen by comparing the gap values of high- $T_{C}$ superconductors measured by this method and by tunneling spectroscopy [8] as well as by the photoemission technique [9]. The contact sizes varied within $5-70 \mathrm{~nm}$. The standard modulation technique was used to register the $d V / d I(V)$ characteristics.

Figure 1 shows the typical PC spectra of the $\operatorname{PrAg}{ }_{6} \operatorname{In}_{6}$ compound measured at different temperatures, which furnish direct evidence for superconductivity in this material. Indeed, as is well known, the resistance decrease of metallic contacts near zero bias voltage is due to the Andreev reflection of quasiparticles, which is always occurring at an $\mathrm{N}-\mathrm{S}$ interface. Thus, the zero-bias resistance minimum which first arises on the $3 \mathrm{~K}$ curve and whose amplitude then increases with lowering temperature (Fig. 1) is a distinct indication of probing the superconducting region. Such indications of superconductivity with a critical temperature $T_{c}$ varying within $1.7-3.3 \mathrm{~K}$ were

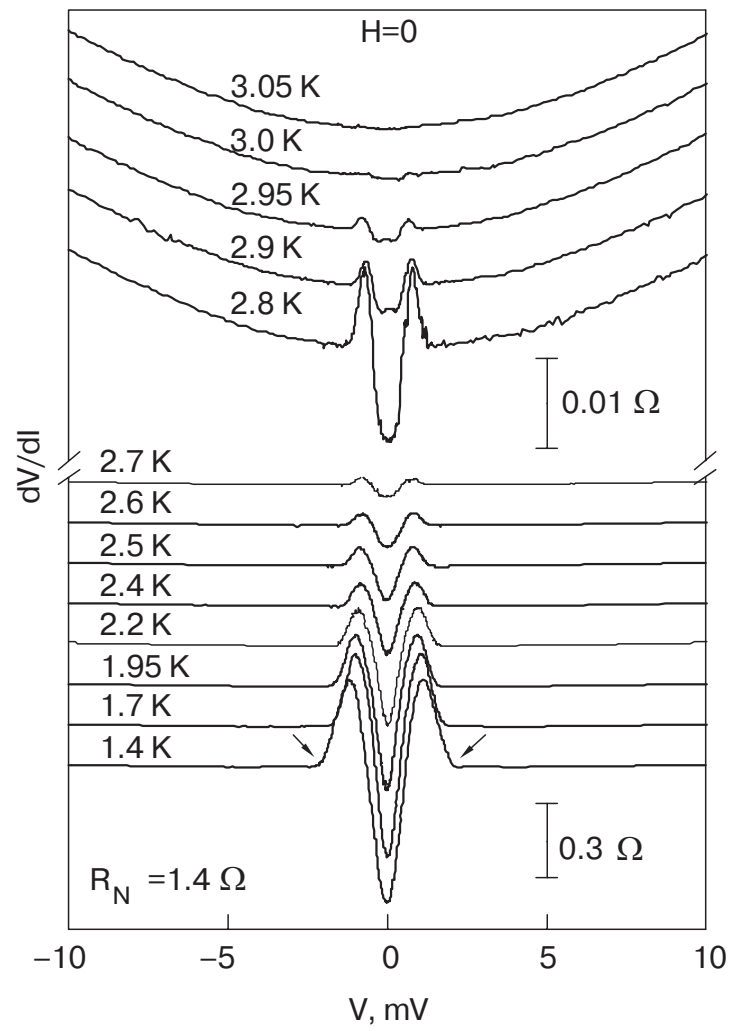

Fig. 1. $d V / d I(V)$ characteristics of the point contact $\mathrm{Ag}^{-}$ $\operatorname{PrAg}_{6} \mathrm{In}_{6}$ measured at different temperatures indicated at each curve. For clarity, the curves are shifted vertically and the upper part of the spectra is shown on an enlarged scale. found in many parts of the fractured sample surface (sometimes, up to $10 \%$ of the total surface). Noticeably, the radio-frequency experiments carried out on the same sample resulted in $T_{c} \sim 8 \mathrm{~K}$ in the first measurements and in the irregular reduction of $T_{c}$ in following tests. Perhaps, the reason is connected with some variations of magnetic structure when the AFM transitions happen. The critical magnetic field could reach about $0.5 \mathrm{~T}$ in some cases, which essentially excluded the possibility of superconductivity arising in the In clusters, which could appear, for instance, due to composition variations. Then, if the superconducting features in our spectra were caused by the Andreev reflection from In clusters, the shape of spectra would agree with that typical for $\mathrm{N}-\mathrm{S}$ contacts based on conventional superconductors [10]. Because such spectra were never observed, this is reliable proof that superconductivity in $\mathrm{PrAg}_{6} \mathrm{In}_{6}$ is not due to a free In component. Moreover, the In clusters should result in wide variations of gap-feature voltages in different contacts [11], and that was not observed as well.

The PC spectra have very unusual shape as compared to the conventional BCS superconductors and show a number of striking features. When the temperature goes down, the $d V / d I(V)$ characteristic develops into a triangular-shaped structure at low voltages. This behavior is not consistent with the fully gapped Fermi surface expected for conventional BCS superconductors and may be taken as an indication of the presence of nodes or lines of nodes in the gap function. Indeed, according to the Blonder-Tinkham-Klapwijk (BTK) theory [10] of $\mathrm{N}-\mathrm{S}$ contacts based on conventional superconductors, near $V=0$ the PC spectra should display either a double minimum structure if a potential barrier occurs at the $\mathrm{N}-\mathrm{S}$ boundary or a flat bottom if this barrier is absent. The spectra measured are characterized by the horn structure which is often observed in low-ohmic contacts although its origin is not understood fully even for conventional superconductors. Besides, it is seen that the width of the superconducting structure does not change strongly with temperature and remains nonzero up to $T=3.05 \mathrm{~K}$, where no structure can be seen at all. Because of the extremely unusual type of PC spectra measured, the standard BTK model cannot be used for finding the gap parameter. Hence, the spectra may be characterized only phenomenologically. Each curve is described by the depth of the resistance minimum $R_{0}=R_{N}-R_{S}$, where $R_{N}$ and $R_{S}$ are the contact resistances at $V=0$ in the normal and superconducting states, respectively, and by the width of the zero-bias minimum at its half depth, $2 \delta$.

As is seen in Figs. 1 and 2, with rising temperature the resistance parameter $R_{0}$ decreases and slowly ap- 
proaches zero at $3.05 \mathrm{~K}$. Correspondingly, the relative parameter $R_{S}$ increases and goes to $R_{N}$. Such behavior does not agree with the BTK theory (see inset in Fig. 2). Both of these dependences change their slopes near $2.8 \mathrm{~K}$ showing that the amplitude variations are about $98 \%$ below this temperature and only about $2 \%$ above it. Near $T=2.8 \mathrm{~K}$, the $R_{S}(T)$ curve looks very much like the smeared resistive $\mathrm{N}-\mathrm{S}$ transition. Therefore, we may quite reasonably take this temperature as the critical temperature $T_{c}$. In the vicinity of and above $2.8 \mathrm{~K}$, the behavior of the $R_{0}(T)$ and $R_{S}(T)$ dependences is probably determined by superconducting fluctuations, i.e., Cooper pairs arising above $T_{c}$. It is worth noting that the width of the whole superconducting $d V / d I(V)$ structure (see arrows on the $1.4 \mathrm{~K}$ curve in Fig. 1) and the distance between horns decrease slowly (by $\sim 40 \%$ ) as the temperature increases up to $2.8 \mathrm{~K}$ and then remain practically unchanged in the temperature range from 2.8 to $3.05 \mathrm{~K}$.

The width $2 \delta$ of the resistance minimum has another remarkable temperature dependence. When the temperature increases, $2 \delta$ first decreases (by $\sim 25 \%$ ) but then, near $T_{c}$, comes back practically to its initial value, thus passing through a minimum near $2.2 \mathrm{~K}$. The $\delta$ parameter of the $d V / d I(V)$ structure is usually associated with the energy gap or order parameter in superconductors. On the other hand, the resistance parameters $R_{0}$ and $R_{S}$ are associated with the number of superconducting quasiparticles involved in the Andreev-reflection processes. So, it is reasonable to suppose that near $2.8 \mathrm{~K}$ we see the smooth transformation of the superconducting gap (or order parameter) into a pseudogap with the same energy scale at this

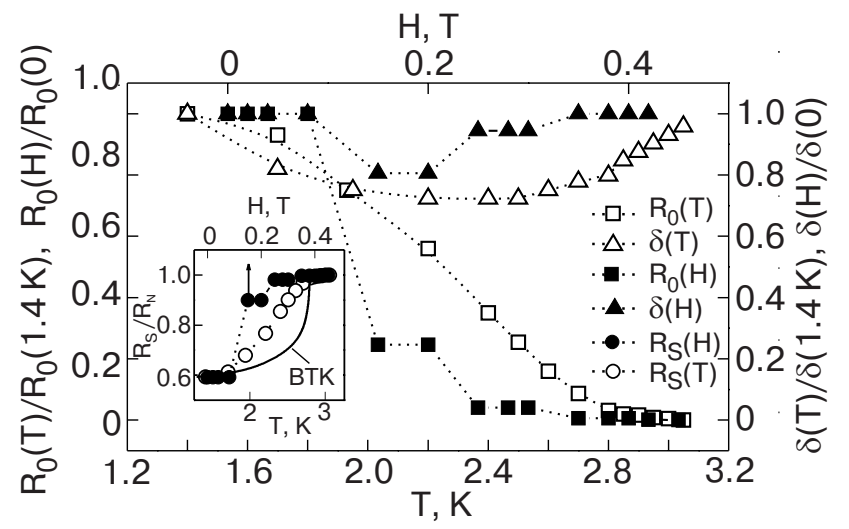

Fig. 2. Temperature and magnetic field dependences of the main parameters for the $d V / d I(V)$ structure: the depth $R_{0}=R_{N}-R_{S}$, and the width $\delta$ of the zero-bias resistance minimum (squares and triangles, respectively) for the contact presented in Figs. 1 and 3. Inset: the analogous dependences of the zero-bias contact resistance $R_{S}$ (circles) together with the dependence expected from BTK theory, $R_{S}(T)$ (solid line).

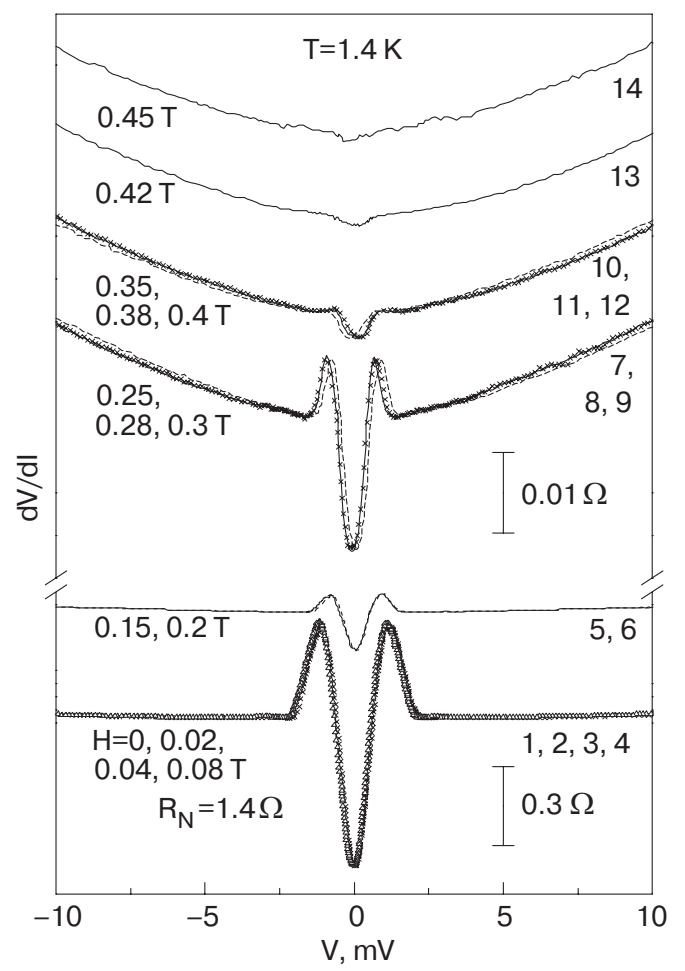

Fig. 3. $d V / d I(V)$ characteristics of the point contact Ag$\operatorname{PrAg}_{6} \mathrm{In}_{6}$ measured at different magnetic fields indicated at each curve. For clarity, the curves are shifted vertically and the upper part of the spectra is shown on an enlarged scale.

temperature, and that only a small amount of fluctuating paired quasiparticles persists in the pseudogap regime.

Our scenario is in qualitative accordance with the results of tunneling spectroscopy of $\mathrm{Bi}_{2} \mathrm{Sr}_{2} \mathrm{CaCu}_{2} \mathrm{O}_{8+\delta}$ single crystals [12], and with the measurements of a coherent boson current in the normal state of the high- $T_{c}$ superconductor $\mathrm{YBa}_{2} \mathrm{Cu}_{3} \mathrm{O}_{y}$ [13]. If the pseudogap model is valid, this may imply that we are dealing with the $d$-wave type of superconductivity proved for high- $T_{c}$ superconductors, for which the pseudogap is known to be typical. In this case the Fermi surface is not gapped entirely and this explains the unusual shape of our $d V / d I(V)$ characteristics. The triangular-shaped PC Andreev-reflection spectra were recently observed by us in $\mathrm{YFe}_{4} \mathrm{Al}_{8}$ [6]. Earlier, similar behavior of the PC spectra was reported in [14] for the heavy-fermion $\mathrm{UPt}_{3}$. This behavior was attributed to the $d$-wave symmetry of the order parameter in the superconductor indicated. The BTK theory extended for $\mathrm{N}-\mathrm{S}$ contacts based on the $d_{x^{2}-y^{2}}$ superconductors also predicts a triangular shape of the PC spectra for most crystallographic directions if the contact barrier transparency is enough high, i.e., the dimensionless barrier strength factor $Z<1$ [15]. 
The effect of the magnetic field on the PC spectra of $\operatorname{PrAg}_{6} \mathrm{In}_{6}$ is very surprising, resulting in the steplike behavior of the spectra in varying field (see Fig. 3 for the same contact as in Fig. 1). Without additional experiments it is difficult to explain this phenomenon clearly. Nonuniform distribution of the superconducting parameters over the sample volume (cf. Figs. 3 and 4) gives evidence for intrinsic structural or/and magnetic inhomogeneities in the given materials, as was earlier seen in contacts with $\mathrm{YFe}_{4} \mathrm{Al}_{8}$ single crystals [6]. In this case, a lot of uncompensated magnetic moments of irregular ordering should appear, which may result in an exotic symmetry of the Cooper pairing and the unconventional behavior of PC spectra in the field.

Remarkably, the main tendencies in magnetic field dependences of the resistance parameters, characterizing the Andreev-reflection structure, remain practically the same as in the case of temperature variations. As is seen in Figs. 2 and 3, the resistance minimum (parameters $R_{0}$ and $R_{S}$ ) vanishes at $H=0.45 \mathrm{~T}$. However, the main changes of the parameters (about 98\%) take place below $0.25 \mathrm{~T}$ (we may take this value as a critical field), showing that only a very small number of paired quasiparticles persist in the field range of $0.25-0.45 \mathrm{~T}$. In this field range, zero-bias minima may be considered as an exhibition of the pseudogap state.

The magnetic field dependence of the energy parameter $\delta$, mirroring the gap or the order parameter, is presented in Fig. 2. Neglecting the steps, one can see that the $\delta(H)$ dependence resembles the $\delta(T)$ one, passing through a minimum within the $0.15-0.20 \mathrm{~T}$ range. Note that the magnetic field dependences of the width of the whole superconducting structure in $d V / d I(V)$ and the distance between the horns, which also correlate with the superconducting gap (order parameter), resemble very much the temperature dependences discussed above.

On some of the point contacts we observed quite interesting behavior of the PC spectra in an applied magnetic field (Fig. 4). In these spectra, after practically full suppression of the low-magnetic-field Andreev-reflection structure typical for $\mathrm{PrAg}_{6} \mathrm{In}_{6}$ (at $0.08 \mathrm{~T}$ for the contact in Fig. 4), there arises another pseudogap-like structure. The latter first increases with field but then disappears in a higher field, slightly above $0.35 \mathrm{~T}$ for the given contact. In some cases this high-field structure could persist up to $H=2.5 \mathrm{~T}$.

The data presented above strongly imply that the arising of the pseudogap state is attributable to superconductivity. Indeed, nothing other than the Andreev reflection associated with fluctuating Cooper pairs can result in the zero-bias resistance minimum on the

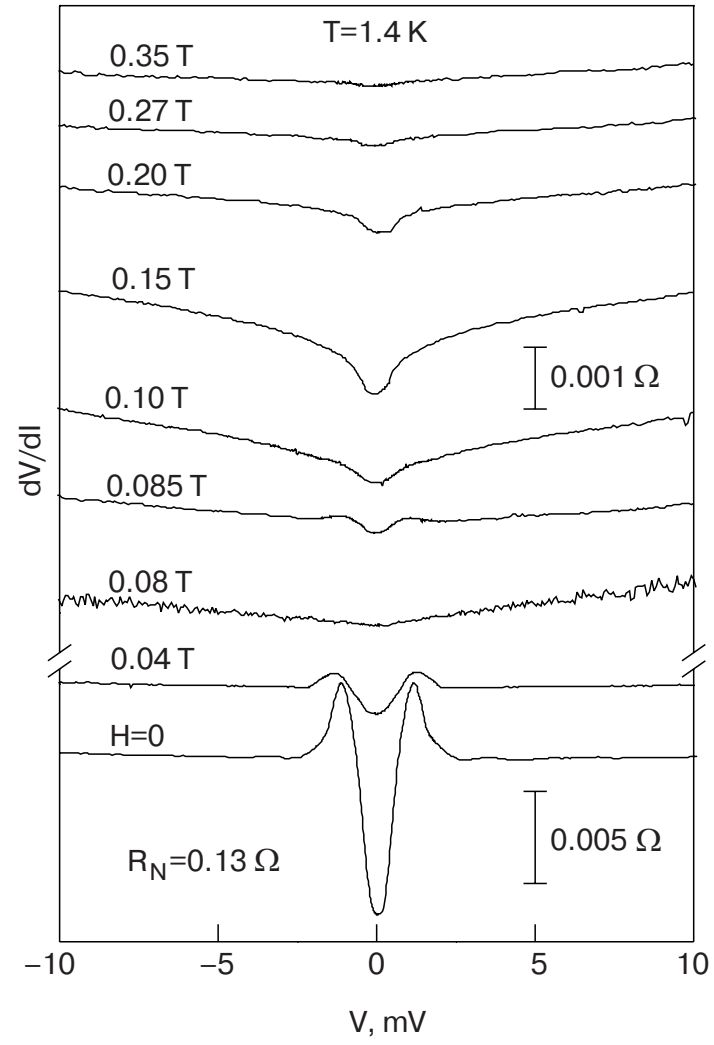

Fig. 4. Another type of $d V / d I(V)$ characteristics for the point contact $\mathrm{Ag}-\mathrm{PrAg}_{6} \mathrm{In}_{6}$ measured at different magnetic fields indicated at each curve. For clarity, the curves are shifted vertically and the upper part of the spectra is shown on an enlarged scale. As is seen, the new pseudogap-like structure arises after practically full disappearance of the superconducting Andreev-reflection structure near $H=0.08 \mathrm{~T}$.

PC spectra above $T_{c}$ or $H_{c 2}$. If the pseudogap were not caused by superconductivity, its sign would be opposite to that observed in our experiments, as was observed earlier in the electron-doped cuprate superconductor $\mathrm{Pr}_{2-x} \mathrm{Ce}_{x} \mathrm{CuO}_{4}$, where the pseudogap was shown to be governed by nonsuperconducting factors [16]. As was shown in [17], the Andreev reflection in the pseudogap state can occur from the phase-incoherent preformed Cooper pairs whose possible existence above $T_{C}$ was suggested by Emery and Kivelson [18].

The effect of magnetic field on the pseudogap structure (see Figs. 3 and 4) indicates that the interior magnetic structure of $\operatorname{PrAg}_{6} \operatorname{In}_{6}$ interacts with preformed pairs above $H_{c 2}$ in some unusual way and that interaction is specified strongly by the variations of magnetic ordering over the sample volume. A closely similar situation was discussed for Bi-2201 crystals of different doping levels [19], which could be compared with the spin variations in our sample. However, at the moment the machanism of this interaction cannot be explained properly. 
In conclusion, direct evidence for superconductivity in the new ternary magnetic compound $\operatorname{PrAg}_{6} \operatorname{In}_{6}$ has been obtained for the first time in point-contact Andreev-reflection experiments. Observation of the pseudogap and step-like structures in the Pr compound is a further (besides the triangular-shaped Andreev features of the same width in different contacts and the high critical magnetic field indicated above) strong proof that the superconductivity cannot be connected with hypothetical chemically free In atoms, but rather originates from the primary crystal structure. The small amount of superconducting phase $(<10 \%$ of total sample volume) may result from some distortions of the crystal structure, e.g., when the slight displacements of magnetic $\mathrm{Pr}$ atoms may provide significant changes of the local magnetic structure. Hereby, the superconducting phase can appear only in separate sample regions with certain magnetic order. The unconventional shape of the point-contact characteristics $d V / d I(V)$ strongly implies non-s-wave symmetry of the Cooper pairing. The basic parameters of the $d V / d I(V)$ structure which undoubtedly are associated with the energy gap or the order parameter in superconductors do not become zero when the temperature or magnetic field increases up to the critical value. Herewith, the superconducting spectra are transformed smoothly into another gap-like structure, demonstrating gradual conversion of the superconducting gap (order parameter) into the pseudogap. This means that between the superconducting and normal states there exists an unusual intermediate (pseudogap) state which possesses properties of both the normal and superconducting phases [20]. According to our knowledge, this is the first observation of the pseudogap regime in a non-high- $T_{c}$ superconductor. Finally, the exciting step-like transitions of the $d V / d I(V)$ Andreev-reflection spectra caused by the magnetic field have been discovered in $\operatorname{PrAg}_{6} \mathrm{In}_{6}$.

We would like to thank G.A. Gogadze for very helpful discussions and V. Zaremba for preparing the $\operatorname{PrAg}{ }_{6} \operatorname{In}_{6}$ samples.
1. A.M. Gurevich, V.M. Dmitriev, V.N. Eropkin, L.A. Ishchenko, N.N. Prentslau, and L.V. Shlyk, Fiz. Nizk. Temp. 25, 15 (1999) [Low Temp. Phys. 25, 10 (1999)].

2. A.M. Gurevich, V.M. Dmitriev, V.N. Eropkin, B.Yu. Kotur, N.N. Prentslau, V. Suski, A.T. Terekhov, and L.V. Shlyk, Fiz. Nizk. Temp. 27, 1308 (2001) [Low Temp. Phys. 27, 967 (2001)].

3. P. Schobinger-Papamantellos, K.H.J. Buschow, and C. Ritter, J. Magn. Magn. Mater. 186, 21 (1998).

4. J.A. Paixão, M.R. Silva, J.C. Waerenborgh, A.P. Gonçalves, G.H. Lander, P.J. Brown, M. Godinho, and P. Burlet, Phys. Rev. B63, 054410 (2001).

5. E. Talik, M.-E. Lucas, W. Suski, and R. Troć, J. Alloys Comp. 350, 72 (2003).

6. V.M. Dmitriev, L.F. Rybaltchenko, P. Wyder, A.G.M. Jansen, N.N. Prentslau, and W. Suski, Fiz. Nizk. Temp. 28, 374 (2002) [Low Temp. Phys. 28, 260 (2002)].

7. V.I. Zaremba, Ya.M. Kalychak, Ya.V. Galadzhun et al., J. Solid State Chem. 145, 216 (1999).

8. J.R. Kirtley, Inter. J. Mod. Phys. 4, 181 (1990).

9. T. Timusk and B. Statt, Rep. Prog. Phys. 62, 61 (1999)

10. G.E. Blonder, M. Tinkham, and T.M. Klapwijk, Phys. Rev. B25, 4515 (1982).

11. O.I. Shklyarevskii, A.M. Duif, A.G.M. Jansen, and P. Wyder, Phys. Rev. B34, 1956 (1986).

12. Ch. Renner, B. Revaz, J.-Y. Genoud, K. Kadowaki, and Ø. Fischer, Phys. Rev. Lett. 80, 149 (1998).

13. K. Kawabata, S. Tsukui, Y. Shono, O. Michikami, H. Saakura, K. Yoshiara, Y. Kakehi, and T. Yotsuya, Phys. Rev. B58, 2458 (1998).

14. Y. De Wilde, J. Heil, A.G.M. Jansen, P. Wyder, R. Deltour, W. Assmus, A. Menovsky, W. Sun, and L. Taillefer, Phys. Rev. Lett. 72, 2278 (1994).

15. Y. Tanuma, Y. Tanaka, and S. Kashiwaya, Phys. Rev. B64, 214519 (2001).

16. A. Biswas, P. Fournier, V.N. Smolyaninova, R.C. Budhani, J.S. Higgins, and R.L. Greene, Phys. Rev. B64, 104519 (2001).

17. H.-Y. Choi, Y. Bang, and D.K. Campbell, cond-mat/9902125 (unpublished).

18. V.J. Emery and S.A. Kivelson, Nature 374, 434 (1995).

19. A.N. Lavrov, Y. Ando, and S. Ono, Europhys. Lett. 57, 267 (2002).

20. A.A. Abrikosov, Phys. Rev. B64, 104521 (2001). 